\title{
On the use of genome-wide data to model and date the time of anthropogenic hybridisation: an example from the Scottish wildcat
}

\author{
Jo Howard-McCombe ${ }^{1}$, Daniel Ward ${ }^{1}$, Andrew Kitchener ${ }^{2}$, Dan Lawson ${ }^{1}$, Helen Senn ${ }^{3}$, and \\ Mark Beaumont ${ }^{1}$ \\ ${ }^{1}$ University of Bristol \\ ${ }^{2}$ National Museums Scotland \\ ${ }^{3}$ Royal Zoological Society of Scotland
}

March 2, 2021

\begin{abstract}
While hybridisation has long been recognised as an important natural phenomenon in evolution, the conservation of taxa subject to introgressive hybridisation from domesticated forms is a subject of intense debate. Hybridisation of the Scottish wildcat, the UK's sole extant native felid, with the domestic cat is a good example in this regard. We develop a modelling framework to determine the timescale and mode of introgression using approximate Bayesian computation (ABC). Applying the model to ddRAD-seq data from 129 individuals, genotyped at 6,546 loci, we show that a population of wildcats genetically distant from domestic cats is still present in Scotland, though these individuals are found almost exclusively within the captive breeding program. Most wild-living cats sampled were introgressed to some extent. Additionally, we evaluate the effectiveness of current methods that are used to classify hybrids. We show that an optimised 35 SNP panel is a better predictor of the ddRAD-based hybrid score in comparison with a morphological method.
\end{abstract}

\section{Hosted file}

Howard-McCombe_MainDocument.pdf available at https://authorea.com/users/398990/articles/ 511574-on-the-use-of-genome-wide-data-to-model-and-date-the-time-of-anthropogenichybridisation-an-example-from-the-scottish-wildcat 